A MODIFIED LOGARITHMIC PROBABILITY GRAPH FOR THE INTERPRETATION OF MECHANICAL ANALYSES

OF SEDIMENTS

BY

GEORGE H. OTTO 


\title{
A MODIFIED LOGARITHMIC PROBABILITY GRAPH FOR THE INTERPRETATION OF MECHANICAL ANALYSES OF SEDIMENTS*
}

\author{
GEORGE H. OTTO \\ Soil Conservation Service, Cooperative Laboratory \\ California Institute of Technology \\ Pasadena, California
}

\begin{abstract}
Mechanical analyses of sediments are studied by comparison with other analyses. Both graphical and numerical methods of comparison are in use, each kind having its limitations.

Two terms of a Gram-Charlier series, expressed in Krumbein's phi units, represent the data in many types of analyses, thus enabling them to be reduced to three numerical values or parameters. Graphical methods are given for recognizing such analyses, for obtaining the parameters, and for evaluating the extent to which they summarize the analytical information. A general procedure is outlined for systematic interpretation of mechanical analyses. The advantages of this method of interpretation are shown by considering in detail the information that can be deduced from the graphical study of a sieve analysis of a dune sand.
\end{abstract}

\section{INTRODUCTION}

Mechanical analyses are studied and evaluated by comparing them either with analyses of type sediments or with those of a related group. Both graphical and numerical methods are employed. The former seek similarities in geometrical form between graphs of two or more analyses. When two or more analyses give the same graph, it is usually inferred that the environment affecting particle diameter composition is essentially the same. Although entire analyses are used in most graphical comparisons, the methods are by no means equally efficient; histograms, for example, not only may fail to disclose pertinent information, but may even suggest erroneous comparisons (10, p. 68).

Numerical methods of comparison (9, 11) attempt to reduce each mechanical analysis to a set of numerical values or parameters that summarize the information. These numerical values may be obtained by computation or by graphical methods. Sets of numerical values are compared with those of type sediments or with others of the same series. Close

* Published with permission of the Soil Conservation Service, U. S. Dept. of Agriculture. numerical agreement between two or more sets is generally taken as evidence of similarity in the environment affecting mechanical composition.

Such methods further presume that the parameters chosen to represent the mechanical analysis are capable of reproducing essentially all information in the analysis except sampling fluctuations. When the parameters are incapable of doing this, potential information is thereby lost. Consequently, it is not correct to infer that the environment affecting mechanical composition is essentially the same merely because a convenient set of parameters happens to have the same numerical values for a series of samples.

Numerical values which contain the essential information in a mechanical analysis may becalled the relevant parameters. ${ }^{1}$ However, interpretation of me-

1 This definition was suggested but not explicitly stated by R. A. Fisher (3, p. 6). Fisher distinguishes between statistics and parameters, the former being used to denote values computed from measurements on samples and the latter to denote the corresponding true values of the parent population from which the samples were drawn. In this paper parameter has its ordinary mathematical meaning. 
chanical analyses is not complete when each analysis has been reduced to relevant parameters which have been compared with each other or with those of type sediments. Complete interpretation includes correlation of the parameters with the physical environment in which the sediment was formed.

\section{MATHEMATICAL PROPERTIES OF MECHANICAL ANALYSES}

Certain mathematical properties of mechanical analyses deserve mention:

1. Particle diameter is a property in which the ratio of change, rather than the amount of change, is fundamental (13, pp. 85-90; 9). Use of a logarithmic measure of diameter such as the phi unit $(10$, p. 76$)$ and the parameters based on it $(9,11)$ avoids many rational difficulties in the interpretation of mechanical analyses.

2. Mechanical analyses are usually expressed in weight percentages instead of number percentages. This fact complicates application of sampling error theory, but does not prevent using statistical methods for condensing the data.

3. A particle has many kinds of diameters $(4,5 ; 17,13$, pp. 93-95; 6), while very different looking particles might correctly be said to have the same diameter.

4. Unless the samples are collected with proper consideration to the mechanism of deposition (14), the size distribution may be complex.

5. Small percentages at both ends of the analysis have physical significance; for instance, one per cent of clay in a fine sand will greatly alter cohesion and plasticity. Consequently, such statistics as the median and quartile deviation (9), which are virtually unaffected by changes at the ends of the distribution, cannot efficiently measure the dependence of these properties on the mechanical composition.

The parameters based on the first few logarithmic moments (11) of a mechanical analysis are computed from the entire analysis and always contain much of the information in it. In order to determine whether they are the relevant parameters, it is usually necessary to know the frequency function or mathematical law which expresses the distribution of particle diameters. Such functions have not yet been derived from consideration of the environment of deposition. However, two terms of a Gram-Charlier series $^{2}$ expressed in phi units, have proved suitable for a wide variety of sediments deposited in relatively simple environments. In such cases the three parameters of this function contain the essential information in the analysis and are thus the relevant parameters. For many other sediments the deviations of their analyses from this frequency function can be accounted for by considering subordinate environmental factors and differences in the mechanical composition of the several layers from which the field sample was taken.

\section{THE PHI PROBABILITY GRAPH}

The phi probability graph is a modification of the logarithmic probability graph invented by Hazen (7) in which cumulative percentage is plotted against the logarithm of the independent vari-

2 In the form adopted in this paper the parameters and limits of integration are expressed entirely in phi units; thus, from the point of view of ordinary units of length, the series is a logarithmic one. It is of the usual type as far as computation and actual reasoning are involved. In the integral form the first two terms may be expressed as follows:

$$
Y=\frac{100}{\sqrt{2 \pi}} \int_{0}^{z} e^{-\frac{1}{2} z^{2}} d z-\frac{100 k_{\phi}}{6 \sqrt{2 \pi}}\left[1-\left(1-z^{2}\right) e^{-\frac{1}{2} z^{2}}\right]
$$

where $Y=$ cumulative percentage (area) between the phi arithmetic mean diameter and diameter $\phi$.

$$
z=\frac{\phi-M_{\phi}}{\sigma_{\phi}}
$$

$M_{\phi}=$ phi arithmetic mean diameter.

$\sigma_{\phi}=$ phi standard deviation.

$k_{\phi}=$ phi skewness $=$ Krumbein's $\alpha_{3}$ or twice his $S k_{\phi}(11$, p. 40$)$.

$\phi=$ any diameter defined by the expression $\phi=-\log _{2} \xi$, where $\xi$ is the diameter in millimeters. 


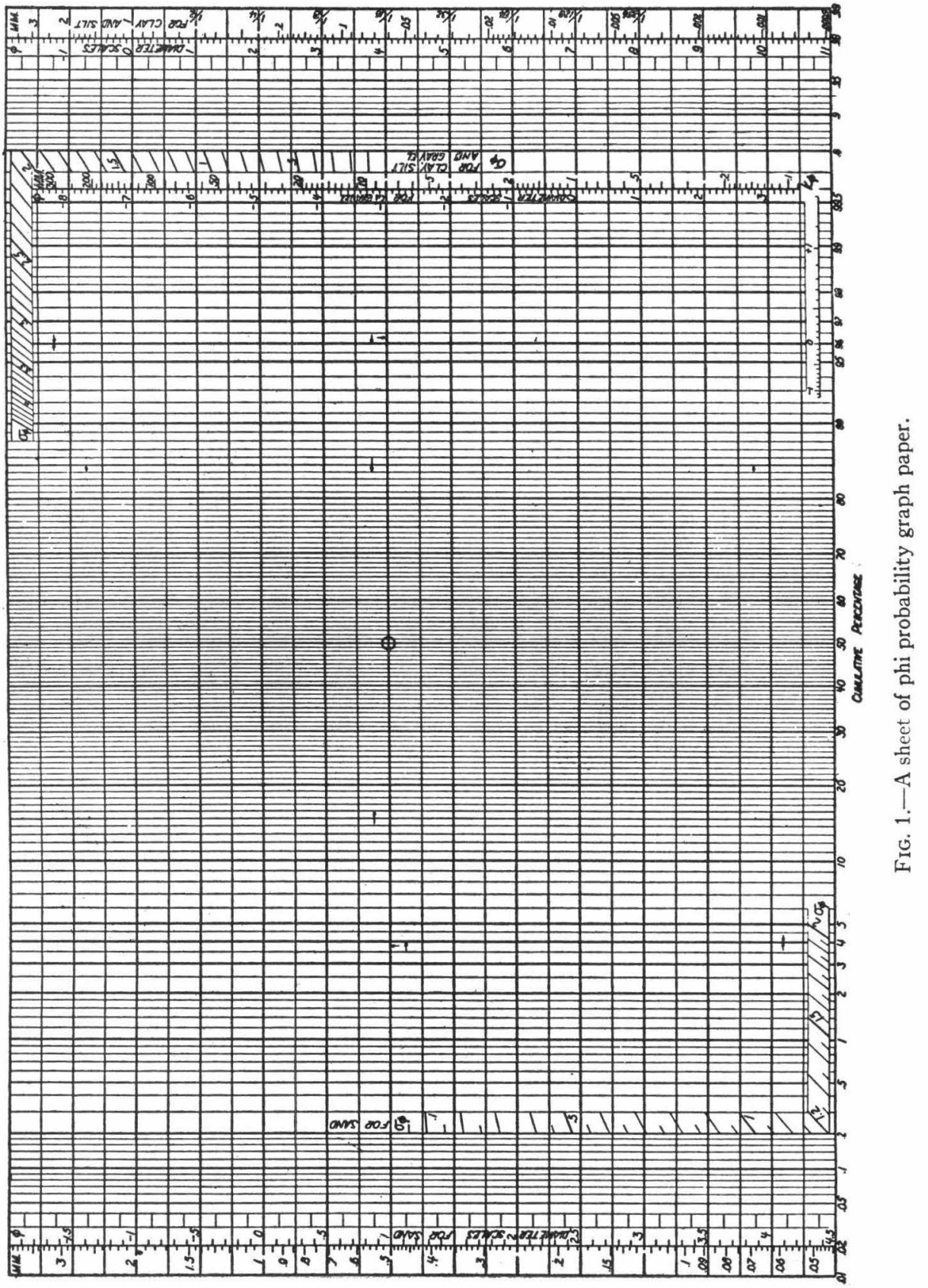


able. Such graphs have been used for several kinds of mechanical analyses (8; 13 , p. $189 ; 6 ; 4)$. On phi probability graph paper, the phi scale of diameter replaces the logarithmic scale; extra scales are added for obtaining certain parmaeters directly in phi terms. Such graphs without the parameter scales have been used by Krumbein (12) and Rouse (16, p. 349) for analyses which plot as straight lines. This paper extends the use of phi-probability graphs to other types of mechanical analyses. A method of constructing the graph paper, together with mathematical proofs, is given in the appendix.

Figure 1 shows a plain sheet of the graph paper. To accommodate the wide range in particle diameter of natural sediments, three sets of diameter scales have been placed on the same sheet, two of which possess a diameter interval twice as large as the third. Thus, the scales marked DIAMETER SCALES $F O R S A N D$ are used for sediments whose particles range from $.044 \mathrm{~mm}$ to 4 $\mathrm{mm}$. The scales labelled DIAMETER SCALES FOR CLAY AND SILT extend from $1 / 2048 \mathrm{~mm}$ to $4 \mathrm{~mm}$; those labelled DIAMETER SCALES FOR GRA VEL extend from $1 / 16 \mathrm{~mm}$ to 512 $\mathrm{mm}$.

Horizontal lines on the graph correspond to grade sizes in common use by geologists and engineers in the United States. The interval between lines corresponding to the left-hand scales is $\frac{1}{4} \phi$. The openings of the widely used Tyler sieves possess dimensions of very nearly $\frac{1}{4} \mathrm{n} \phi$, where $\mathrm{n}$ is an integer, so that the horizontal lines closely approximate sieve sizes. For either right-hand set of scales, the interval between horizontal lines is $\frac{1}{2} \phi$. If the grade-size boundaries come at $\frac{1}{4} \phi$ intervals, intermediate lines are drawn as needed by connecting the pairs of short guide lines located alongside the .03 and 99.97 per cent vertical lines. Cumulative percentages are shown by the vertical lines of the graph spaced according to the probability scale at the bottom of the graph.

\section{PLOTTING THE ANALYSIS}

Plotting the analysis is expedited if the data are arranged as in table 1 . This analysis is plotted on Fig. 2, where, for simplicity, only those scales are shown

TABLE 1. Sieve analysis of dune sand from Palm Springs, California

\begin{tabular}{|c|c|c|c|}
\hline 1 & 2 & 3 & 4 \\
\hline $\begin{array}{c}\text { Diameter of } \\
\text { sieve } \\
\text { openings }\end{array}$ & $\begin{array}{l}\text { Equiva- } \\
\text { lent phi } \\
\text { diam- } \\
\text { eter }\end{array}$ & $\begin{array}{l}\text { Percent- } \\
\text { age re- } \\
\text { tained } \\
\text { on sieve }\end{array}$ & $\begin{array}{l}\text { Per- } \\
\text { centage } \\
\text { finer }\end{array}$ \\
\hline $\begin{array}{l}1.65 \mathrm{~mm} \\
1.17 \\
.833 \\
.589 \\
.417 \\
.295 \\
.208 \\
.147 \\
.104 \\
.074 \\
\text { pan }\end{array}$ & $\begin{array}{r}-\quad .75 \\
-\quad .25 \\
.25 \\
.75 \\
1.25 \\
1.75 \\
2.25 \\
2.75 \\
3.25 \\
3.75\end{array}$ & $\begin{array}{r}.07 \\
.43 \\
5.4 \\
14.5 \\
23.5 \\
27.6 \\
15.6 \\
7.1 \\
2.7 \\
3.1\end{array}$ & $\begin{array}{c}100.00 \\
99.93 \\
99.5 \\
94.1 \\
79.6 \\
56.1 \\
28.5 \\
12.9 \\
5.8 \\
3.1\end{array}$ \\
\hline
\end{tabular}

which are used in the study of the analysis; many percentage lines are also omitted. The procedure is as follows:

Find in column 4 the percentage 3.1 corresponding to the smallest sieve diameter, $.074 \mathrm{~mm}(\phi=3.75)$, in column 1 of table 1 . Select the horizontal line on the graph corresponding to $\phi=3.75$ and locate the circle one-fifth of the distance between the 3.0 and 3.5 per cent lines. Locate the next percentage, 5.8 , on the line $\phi=3.25$. Since in this analysis the ratio interval between sieves is constant and equal to $\frac{1}{2} \phi$, place each succeeding percentage on the next unaccented line. (The 100 per cent point cannot be plotted, which is in accord with the fact that a larger sample generally has still larger particles.) Fit a smooth curve to the data, preferably first connecting the points with straight line segments. In fitting the curve, remember that points based on fewer than 20 particles in a grade are subject to large sampling errors and that for fine grades in amounts less than .5 per cent the laboratory technique is seldom good enough to benefit from 


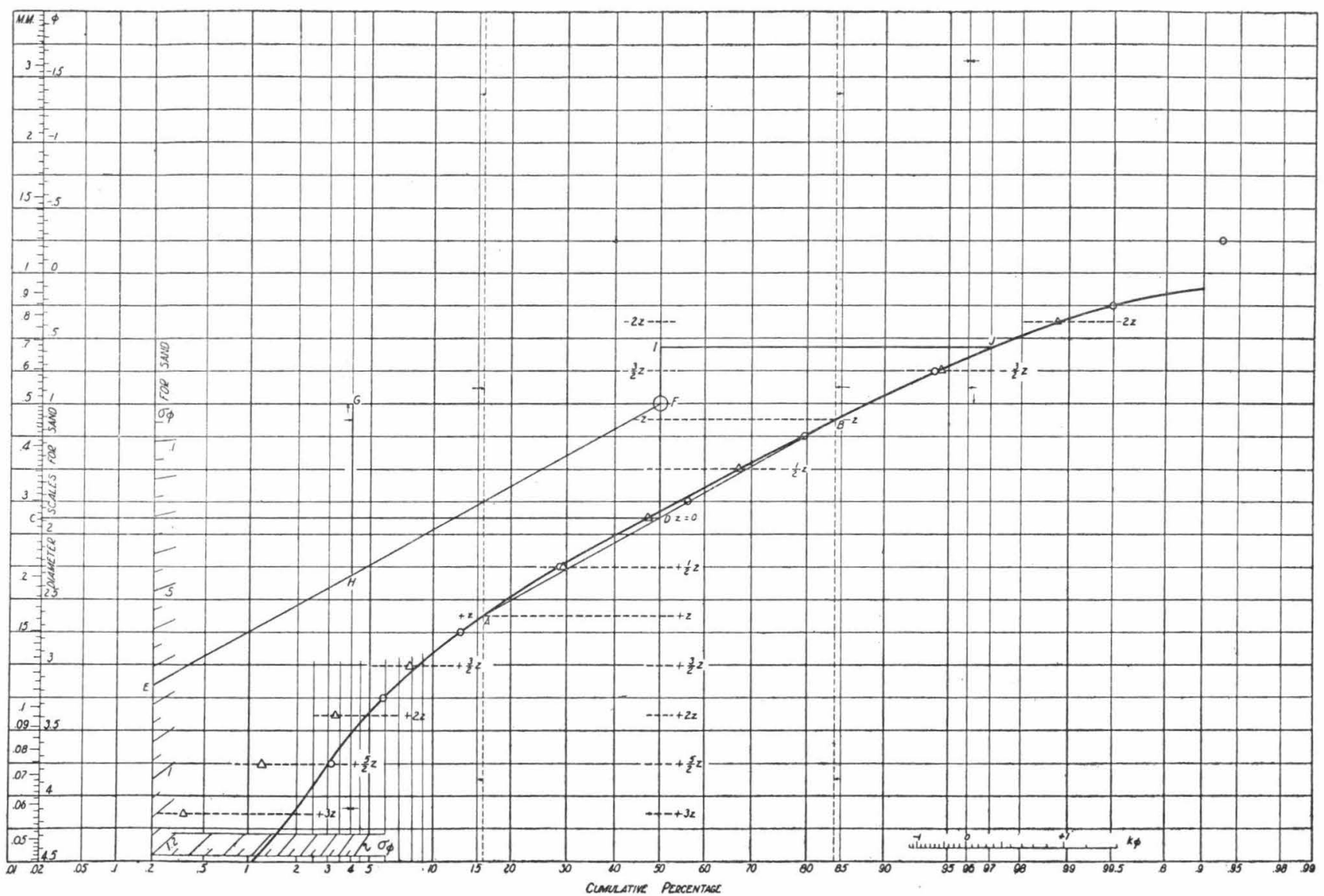

FIG. 2.-A sieve analysis of dune sand from Palm Springs, California, plotted on phi probability graph paper. The steps in the graphical determination of parameters and construction of the theoretical curve are shown. 
the large number of particles actually present. In general, the fitted curve may depart from any point by an amount as large as the combined laboratory errors.

The phi probability graph greatly reduces curvature in graphs of most analyses, as shown by contrasting the conventional plot in Fig. 3 with that in Fig. 2. Extrapolation is less uncertain, both for this reason and because the small percentages at both ends of the analysis are represented with an accuracy equal to the best analytical technique.

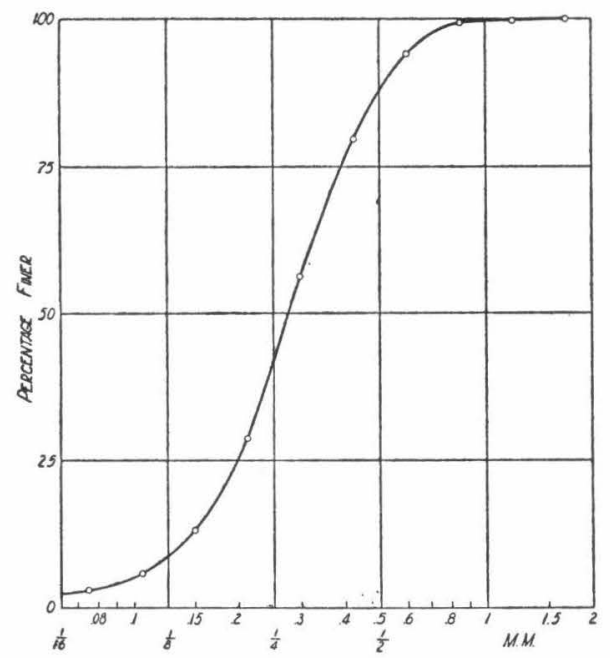

FIG. 3.-Semi-logarithmic graph of same analysis shown in Figure 2.

If the fitted curve resembles any curve in Fig. 4, it is probable that the twoterm series given on page 63 summarizes the information in the analysis. The numerical values of the parameters may then be obtained graphically; but these values, especially the skewness, should not be accepted without fitting the theoretical curve as explained later. If the curves do not resemble any in Fig. 4, proceed to the section, SYSTEMATIC INTERPRETATION OF A NAL YSES. (Curves A and B of Fig. 5 are common types not represented by two terms of a Gram-Charlier series.)

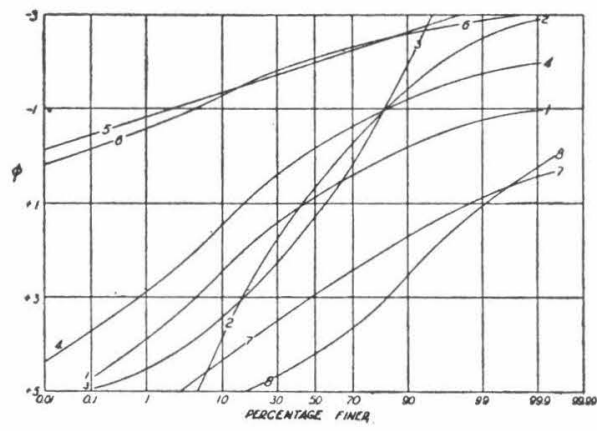

FIG. 4.-Graphs of a two-term GramCharlier series showing effect of changes in the parameters.

$\begin{array}{cccccccc}\text { Curve } & M_{\phi} & \sigma_{\phi} & k_{\phi} & \text { Curve } & M_{\phi} & \sigma_{\phi} & k_{\phi} \\ 1 & 1 & 1 & +1 & 5 & -2 & .5 & 0 \\ 2 & 1 & 2 & +1 & 6 & -2 & .5 & +1 \\ 3 & 1 & 2 & -1 & 7 & 3 & 1 & \pm .3 \\ 4 & 0 & 1 & +1 & 8 & 4 & 1 & -1.2\end{array}$

GRAPHICAL DETERMINATION OF PARAMETERS

To obtain $\mathrm{M}_{\phi}$ : Draw a straight line $A B$ (fig. 2) connecting the intersections of the fitted curve and the 15.9 and 84.1 per cent dotted lines. These lines are identified by three small horizontal arrows. Where the 50 per cent line crosses the line $A B$, draw a horizontal line $C D$ intersecting the diameter scale used in plotting the analysis. Read off the value of $\mathrm{M}_{\phi}, 1.88 \phi$, on the arithmetic phi scale and the corresponding geometric mean diameter, $.272 \mathrm{~mm}$, from the other side of the intersection.

To obtain $\sigma_{\phi}$ : Draw a line $E F$ parallel to line $A B$ through the center of the small circle near the middle of the graph. Extend the line $E F$ to the phi standard deviation scale on the same side of the graph as the scale used for plotting the analysis. The phi standard deviation scales are labelled $\sigma_{\phi}$ and are the slant-line scales along the upper right and lower left corners of the graph. Read the value of $\sigma_{\phi}, .75 \phi$, at $E$ at the intersection of the line $E F$ with the scale. The lower left scale labelled FOR SA ND is graduated in intervals of $.05 \phi$ and the other scale (see fig. 1) in intervals of $.1 \phi$.

To obtain $k_{\phi}$ : Set a pair of dividers to 

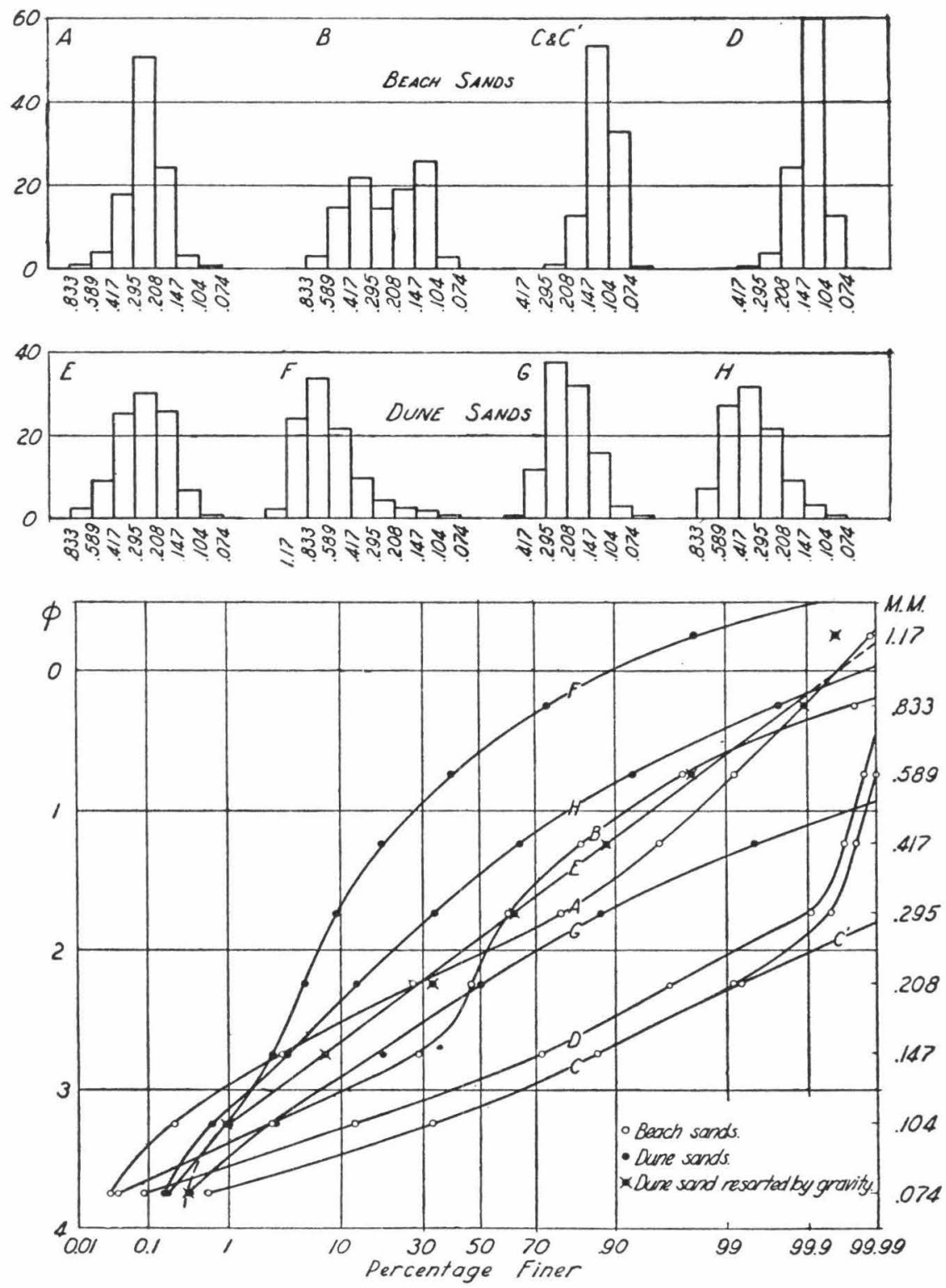

FIG. 5.-Graphical comparison of sieve analyses typical of beach and dune sands.

Location of Samples

A. West of Dune Point, Los Angeles County, California.

B. Daytona Beach, Florida. Sample collected following several days of offshore winds.

C. North end of Mustang Island, Texas, at Aransas Pass.

$C^{\prime}$. Same sample after removing shells with dilute acid.

D. Eight miles South of Aransas Pass, Mustang Island, Texas.

E. Opposite highway bridge over Whitewater Wash, North of Palm Springs, California.

F. Ripple mark crests in the lee of a barchane in the dune complex West of Yuma, California.

$\mathrm{G}$. Lee slope of above barchane.

H. Crest of active dune area near Oxnard, California. 
the length $G H$ on the vertical 4 per cent line between its intersection with the line $E F$ and the horizontal line passing through the circle at the middle of the graph. The point $G$ is indicated by two small arrows at right angles to each other. When $\sigma_{\phi}$ is read from the right hand scale (Fig. 1), use the 96 per cent line instead of the 4 per cent line; the point corresponding to $G$ is indicated as before. Next, lay off the distance $D I$, equal to $G H$, on the 50 per cent line starting at its intersection with the line $A B$. From point $I$ draw a horizontal line $I J$ intersecting the fitted curve. Read the percentage, 97.1 , at the intersection. On the $k_{\phi}$ scale near the lower right corner of the graph read the value of the skewness, .36 , corresponding to the percentage just obtained.

The algebraic sign of the skewness depends on what is assumed to constitute a negative deviation from the phi arithmetic mean. The convention used by Krumbein $(11$, p. 40$)$ is adopted here: those phi values algebraically larger than $\mathbf{M}_{\phi}$ or the assumed mean are considered positive; similarly, that part of the distribution coarser than $M_{\phi}$ is considered negative. If a straight line fits the plotted points, the skewness is zero. In this case the fitted straight line takes the place of the line $A B$. The remaining procedure for obtaining $M_{\phi}$ and $\sigma_{\phi}$ is the same as described above.

\section{GRAPHICAL CONSTRUCTION OF THE THEORETICAL ANALYSIS}

In the previous section, it was assumed that two terms of a GramCharlier series form an adequate frequency function for representing the mechanical analysis. If this assumption is correct, the theoretical curve computed from these parameters should coincide with the curve drawn through the observed points. The amount of divergence thus furnishes a qualitative estimate of fit. Computation of the theoretical curve is tedious, however, and the results so obtained are not here suitable for applying the quantitative Chi Square test of goodness of fit (1, pp. 264-267), because mechanical analyses are expressed in weight percentages. A rapid graphical method was therefore developed for comparing the theoretical analysis with the observed data. Either calculated or graphically obtained values of the parameters may be used.

If calculated values are used, plot the analysis on phi probability graph paper and draw a horizontal line intersecting the curve at the position of the phi arithmetic mean diameter and label this line $z=O$. This line coincides with the line $C D$ when the parameters have been obtained graphically. Moreover, the vertical distance between $A$ and $B$ in Fig. 2, when laid off along the proper phi scale starting at $\phi=0$, corresponds numerically to $2 \sigma_{\phi}$. Consequently, to obtain a distance equal to $\sigma_{\phi} / 2$, set a pair of dividers to a length on the phi scale corresponding to one-half the numerical value of $\sigma_{\phi}$ or to one-fourth the vertical distance between $A$ and $B$. Now set one point of the dividers where the line, $z=O$, intersects the 50 per cent line; lay off successive intervals of the distance corresponding to $\sigma_{\phi} / 2$ along the 50 per cent line. These distances correspond to intervals of $\frac{1}{2} z$ in the GramCharlier equation. Label the points as indicated in Fig. 2: $-\frac{1}{2} z,-z,-3 / 2 z$, $-2 z$, etc. above the line $z=O$ and $+\frac{1}{2} z,+z,+3 / 2 z,+2 z$, etc. below the line $z=0$. Draw horizontal lines intersecting the plotted curve and passing through these points.

Table 2 gives the corresponding cumulative percentages of the theoretical curve for skewness intervals ${ }^{3}$ of $.1 k_{\phi}$. Select the column corresponding most closely to the value of $k_{\phi}$. Thus if $k_{\phi}=.36$, select the column marked $k_{\phi}=.4$. Starting with the lowest percentage given in that column, plot this percentage on the horizontal line (fig. 2) whose $z$ value is the same as that given in the table. Plot the next higher percentage in the table

${ }^{3}$ An interval of .1 was adopted because repeated analyses of the same field sample often show larger deviations. 
TABLE 2. Cumulative percentages of the theoretical analyses based on two terms of a Gram-Charlier series

\begin{tabular}{|c|c|c|c|c|c|c|c|c|c|c|c|c|c|}
\hline \multirow{2}{*}{$z$} & \multicolumn{12}{|c|}{$k_{\phi}$} & \multirow[b]{2}{*}{$z$} \\
\hline & +.1 & +.2 & +.3 & +.4 & +.5 & +.6 & +.7 & +.8 & +.9 & +1.0 & +1.1 & +1.2 & \\
\hline $\begin{array}{l}-3 . \\
-2.5 \\
-2 . \\
-1.5 \\
-1.5 \\
-.5\end{array}$ & $\begin{array}{l}99.924 \\
99.53 \\
98.00 \\
93.59 \\
84.1 \\
68.7\end{array}$ & $\begin{array}{l}99.983 \\
99.69 \\
98.26 \\
93.86 \\
84.1 \\
68.3\end{array}$ & $\begin{array}{l}99.839 \\
98.54 \\
94.13 \\
84.1 \\
67.8\end{array}$ & $\begin{array}{l}98.80 \\
94.40 \\
84.1 \\
67.4\end{array}$ & $\begin{array}{l}99.08 \\
94.67 \\
84.1 \\
66.9\end{array}$ & $\begin{array}{l}99.34 \\
94.94 \\
84.1 \\
66.5\end{array}$ & $\begin{array}{l}99.62 \\
95.21 \\
84.1 \\
66.1\end{array}$ & $\begin{array}{l}99.885 \\
95.48 \\
84.1 \\
65.6\end{array}$ & $\begin{array}{l}95.75 \\
84.1 \\
65.2\end{array}$ & $\begin{array}{l}96.02 \\
84.1 \\
64.7\end{array}$ & $\begin{array}{l}96.29 \\
84.1 \\
64.3\end{array}$ & $\begin{array}{l}96.56 \\
84.1 \\
63.9\end{array}$ & $\begin{array}{l}-3 . \\
-2.5 \\
-2.5 \\
-1.5 \\
-1.5 \\
-.5\end{array}$ \\
\hline 0 & 49.3 & 48.7 & 48.0 & 47.3 & 46.7 & 46.0 & 45.3 & 44.7 & 44.0 & 43.4 & 42.7 & 42.0 & 0 \\
\hline $\begin{array}{l}+.5 \\
+1 . \\
+1.5 \\
+2 . \\
+2.5 \\
+3 . \\
+3.5 \\
+4 .\end{array}$ & $\begin{array}{c}30.4 \\
15.9 \\
6.95 \\
2.54 \\
.77 \\
.194 \\
.039 \\
.006 \\
-.1\end{array}$ & $\begin{array}{c}30.0 \\
15.9 \\
7.22 \\
2.82 \\
.93 \\
.25 \\
.056 \\
.010 \\
-.2\end{array}$ & $\begin{array}{c}29.5 \\
15.9 \\
7.49 \\
3.08 \\
1.08 \\
.31 \\
.072 \\
.013 \\
-.3\end{array}$ & $\begin{array}{c}29.1 \\
15.9 \\
7.76 \\
3.36 \\
1.23 \\
.37 \\
.088 \\
.016 \\
-.4\end{array}$ & $\begin{array}{c}28.7 \\
15.9 \\
8.03 \\
3.62 \\
1.39 \\
.43 \\
.105 \\
.021 \\
-.5\end{array}$ & $\begin{array}{c}28.2 \\
15.9 \\
8.30 \\
3.90 \\
1.54 \\
.49 \\
.122 \\
.024 \\
-.6\end{array}$ & $\begin{array}{c}27.8 \\
15.9 \\
8.57 \\
4.16 \\
1.70 \\
.55 \\
.138 \\
.027 \\
-.7\end{array}$ & $\begin{array}{c}27.3 \\
15.9 \\
8.84 \\
4.44 \\
1.85 \\
.61 \\
.154 \\
.030 \\
-.8\end{array}$ & $\begin{array}{c}26.9 \\
15.9 \\
9.11 \\
4.70 \\
2.00 \\
.67 \\
.171 \\
.034 \\
-.9\end{array}$ & $\begin{array}{c}26.5 \\
15.9 \\
9.38 \\
4.98 \\
2.16 \\
.73 \\
.187 \\
.037 \\
-1.0\end{array}$ & $\begin{array}{c}26.0 \\
15.9 \\
9.65 \\
5.24 \\
2.31 \\
.78 \\
.20 \\
.040 \\
-1.1\end{array}$ & $\begin{array}{c}25.6 \\
15.9 \\
9.92 \\
5.52 \\
2.46 \\
.84 \\
.22 \\
.044 \\
-1.2\end{array}$ & $\begin{array}{l}+.5 \\
+1.5 \\
+1.5 \\
+2.5 \\
+2.5 \\
+3.5 \\
+3.5 \\
+4 .\end{array}$ \\
\hline $\begin{array}{l}-4 . \\
-3.5 \\
-3.5 \\
-2.5 \\
-2.5 \\
-1.5 \\
-1.5 \\
-\quad .5\end{array}$ & $\begin{array}{l}99.994 \\
99.961 \\
99.806 \\
99.23 \\
97.46 \\
93.05 \\
84.1 \\
69.6\end{array}$ & $\begin{array}{l}99.990 \\
99.944 \\
99.75 \\
99.07 \\
97.18 \\
92.78 \\
84.1 \\
70.0\end{array}$ & $\begin{array}{l}99.987 \\
99.928 \\
99.69 \\
98.92 \\
96.92 \\
92.51 \\
84.1 \\
70.5\end{array}$ & $\begin{array}{l}99.984 \\
99.912 \\
99.63 \\
98.77 \\
96.64 \\
92.24 \\
84.1 \\
70.9\end{array}$ & $\begin{array}{l}99.979 \\
99.895 \\
99.57 \\
98.61 \\
96.38 \\
91.97 \\
84.1 \\
71.3\end{array}$ & $\begin{array}{l}99.976 \\
99.878 \\
99.51 \\
98.46 \\
96.10 \\
91.70 \\
84.1 \\
71.8\end{array}$ & $\begin{array}{l}99.973 \\
99.862 \\
99.45 \\
98.30 \\
95.84 \\
91.43 \\
84.1 \\
72.2\end{array}$ & $\begin{array}{l}99.970 \\
99.846 \\
99.39 \\
98.15 \\
95.56 \\
91.16 \\
84.1 \\
72.7\end{array}$ & $\begin{array}{l}99.966 \\
99.829 \\
99.33 \\
98.00 \\
95.30 \\
90.89 \\
84.1 \\
73.1\end{array}$ & $\begin{array}{l}99.963 \\
99.813 \\
99.27 \\
97.84 \\
95.02 \\
90.62 \\
84.1 \\
73.5\end{array}$ & $\begin{array}{l}99.960 \\
99.80 \\
99.22 \\
97.69 \\
94.76 \\
90.35 \\
84.1 \\
74.0\end{array}$ & $\begin{array}{l}99.956 \\
99.78 \\
99.16 \\
97.54 \\
94.48 \\
90.08 \\
84.1 \\
74.4\end{array}$ & $\begin{array}{l}-4 . \\
-3.5 \\
-3.5 \\
-2.5 \\
-2.5 \\
-1.5 \\
-1.5 \\
-.5\end{array}$ \\
\hline 0 & 50.7 & 51.3 & 52.0 & 52.7 & 53.3 & 54.0 & 54.7 & 55.3 & 56.0 & 56.6 & 57.3 & 58.0 & 0 \\
\hline $\begin{array}{l}+. .5 \\
+1 . \\
+1.5 \\
+2.5 \\
+2.5 \\
+3 .\end{array}$ & $\begin{array}{c}31.3 \\
15.9 \\
6.41 \\
2.00 \\
.47 \\
.076\end{array}$ & $\begin{array}{c}31.7 \\
15.9 \\
6.14 \\
1.74 \\
.31 \\
.017\end{array}$ & $\begin{array}{r}32.2 \\
15.9 \\
5.87 \\
1.46 \\
.161\end{array}$ & $\begin{array}{c}32.6 \\
15.9 \\
5.60 \\
1.20\end{array}$ & $\begin{array}{r}33.1 \\
15.9 \\
5.33 \\
.92\end{array}$ & $\begin{array}{r}33.5 \\
15.9 \\
5.06 \\
.66\end{array}$ & $\begin{array}{r}33.9 \\
15.9 \\
4.79 \\
.38\end{array}$ & $\begin{array}{c}34.4 \\
15.9 \\
4.52 \\
.115\end{array}$ & $\begin{array}{l}34.8 \\
15.9 \\
4.25\end{array}$ & $\begin{array}{l}35.3 \\
15.9 \\
3.98\end{array}$ & $\begin{array}{l}35.7 \\
15.9 \\
3.71\end{array}$ & $\begin{array}{l}36.1 \\
15.9 \\
3.44\end{array}$ & $\begin{array}{l}+.5 \\
+1.5 \\
+1.5 \\
+2.5 \\
+2.5 \\
+3 .\end{array}$ \\
\hline
\end{tabular}


on the next higher $z$ line on the graph, continuing until all the values (shown by triangles in fig. 2) have been plotted.

Compare the theoretical curve corresponding to these points with that obtained from the analysis. The allowable amount of deviation depends on the magnitude of the laboratory errors and of the fluctuation in mathematical form of a series of closely spaced field samples. The fit obtained in Fig. 2 is generally typical of samples from single sedimentation units.

\section{SYSTEMATIC INTERPRETATION OF ANALYSES}

Until frequency functions representing size distribution can be derived mathematically for specific environments, the interpretation of mechanical analyses will necessarily be a comparative study. Consequently, the interpretation of a single analysis is best made by comparing it with analyses of other samples deposited under somewhat different, but definitely known, field conditions. Certain ideal types, which serve as norms of comparison, can generally be deduced. Any deviation therefrom which is greater than sampling and laboratory errors deserves explanation. The interpretation of related analyses, such as those of an environmental pattern study, should involve not only explanation of numerical changes in the parameters, but also changes in mathematical form of the frequency functions that represent the distributions of particle size.

Figure 5 shows nine analyses typical of beach and dune sands. Single samples of beach and dune sands cannot consistently be distinguished from each other by comparing their histograms or ordinary cumulative curves. Because the phi probability graph is sensitive to slight differences in mechanical composition of the end fractions, it is helpful in studying sediments of diverse origin but similar mechanical composition. Each sample used in Fig. 5 was collected from part of a sedimentation unit (14). Curves $F, G$, and $H$ are typical of dune sands low in heavy minerals; such curves are concave downward and generally approach an upper limit of particle diameter when large samples are employed to determine the small percentages in the coarsest grades. Curve $E$ is a micaceous dune sand resorted by a gravity slide on a steep mountain slope; the straight-line tendency is common in sediments sorted by gravity. Curves $A, B, C, C^{\prime}$ and $D$ are typical beach sands. They tend to be concave upward unless there are complications in density and shape of particles, such as in curve $B$, for which the sand consists of rounded quartz grains and platy shell fragments. Curves $C$ and $D$ show notably different histograms, yet the only physical difference between the sediments is a moderate one in average diameter. On the graph this is shown by their similar shape and different position. Curves $C$ and $C^{\prime}$ look alike on the histograms but are readily distinguished on the graph because they differ considerably in skewness. Complete criteria for distinguishing beach and dune sands cannot, of course, be developed from these few analyses alone, for they fail to illustrate many complicating factors, but these curves do give a simplified summary of an extensive study following the plan outlined in the remainder of the paper.

This plan for systematic interpretation was tested on four different environmental patterns, the time required being well repaid by the gain in geological information that otherwise might have remained undiscovered. No assumptions have been made concerning field sampling technique. However, if samples are collected without regard to sedimentation units (14), disappointingly few analyses may be amenable to the mathematical treatment described above. Thus, extremely variable types of analyses are obtained when the samples consist of only a few laminae or a small number of sedimentation units differing greatly in average diameter; on the other hand, when a large number of sedimentation units is included in each 
sample, the mechanical analyses may again show striking uniformity with respect to mathematical type.

The steps recommended for systematic interpretation are as follows:

1. Classify the mechanical analyses according to mode of deposition. For example, in a study of windblown deposits along a desert wash where it leaves the mountains and debouches on the desert floor, sediments such as the following should be distinguished: waterborne sand and gravel in the wash (the source of the windblown deposits), lag sand and gravel, dune sand, dune sand resorted by gravity on steep mountain slopes, rock talus mixed with dune sand, and sandy loess deposited around clumps of vegetation.

2. Plot analyses of typical samples of each kind of sediment on phi probability graph paper, using a single sheet for each sediment type. Wherever possible, plot one or two analyses of sediment deposited under essentially constant conditions and consisting entirely of minerals of the same density and shape characteristics. ${ }^{4}$ These samples may be from any locality, provided the environment of deposition is definitely known to be similar and provided comparable kinds of particle diameter are employed in both sets of analyses.

3. Note whether most of the curves on any one sheet can be classified into one or two distinct geometrical types. Thus, compare the analyses included in each type with analyses of those samples which represent the simplest environment of deposition, and ascertain whether the differences can be assigned qualitatively to specific complicating factors in the environment. In this way, factors affecting mechanical composition are discovered that otherwise might remain unnoticed even with detailed field work.

4. If the curves resemble those in Fig. 4, fit two terms of a Gram-Charlier series to

- Shape is often correlated with particle diameter in sand and gravel deposits; such variations are permissible. The combination of platy and well-rounded grains in the same sample is to be avoided. representative analyses by the methods described above. If the fit is poor or if the curves are obviously different from those in Fig. 4, consider whether the environment of deposition is such that the resulting sediment is actually a mixture of two separate distributions deposited simultaneously or alternately. Sand deposited from a muddy stream is such a sediment, since silt and clay settle out between the sand particles immediately following deposition of the sand; such analyses show a long "tail" of fine sizes. Experiments in progress indicate that the phi probability graph provides a rational quantitative approach to many twocomponent sediments.

5. Plot the remaining analyses on phi probability graph paper and classify the curves according to the types previously deduced. These graphs are conveniently drawn on $8 \frac{1}{2} \times 11$ sheets of arithmetic probability paper, such as Codex \#3127, if a phi scale is laid out along the equispaced rulings; for classification purposes only straight line segments need be fitted to the points. Now indicate the types on a map showing sample locations, also noting any transitional types and highly irregular analyses.

6. Obtain sets of relevant parameters whenever practicable. If only a few analyses are reducible to sets of relevant parameters, compute only those parameters based on the first three moments. Graphically determined parameters are preferable for studies of major factors affecting mechanical composition, because secondary effects are considerably reduced by the curve fitting process and the non-use of the extreme ends of the curve in the graphical constructions. In studies involving correlation of physical properties with mechanical composition, computed parameters are preferable, since they more truly reflect the influence of secondary characteristics of the mechanical analysis. Thus, a "tail" consisting of two per cent silt and clay in a mechanical analysis of fine sand may be unimportant in a study seeking to determine the principal factors controlling average diameter and sorting; yet the 
same amount would considerably affect such a property as porosity.

7. Prepare areal contour maps of the parameters, noting those computed from analyses not satisfactorily represented by the frequency function. In drawing the contours, an erratic value may be ignored if it is reasonably certain that the deviation was caused by defective field sampling. If the parameters consistently fail to form relevant sets, it should be kept in mind that conclusions drawn from the contour maps alone may be erroneous.

8. From the contour maps, and from the map showing locations and types of curves, select one or more sets of analyses which show the change in mechanical composition from the source of the sediment to the highly sorted end products. The analyses in each set should form a logical geographical group. Visual study of these graphs of ten reveals what is happening to the parameters, and as well to the frequency function itself, during the transportation process.

9. Determine how much variation in ap. pearance of the curves and what range in parameter values can reasonably be attributed to combined effects of field-sampling fluctuations and laboratory errors. Fieldsampling fluctuations include cyclical changes too local to be included in a regional investigation and also those fluctuations which remain unpredictable even after numerous closely spaced analyses have been studied. Laboratory errors include those deviations which can be evaluated by successive analyses of the same sample.

A usable estimate of the combined effect of these errors may be obtained from analyses of eight or more samples selected from an area one-fourth as large as that represented by the samples used in the regional study. If the environment changes rapidly in one direction and slowly in another, as along a coastal strip, take the sample from an elongated area or line parallel to the direction of minimum regional change. A set of such samples is required from each distinctly different environment of deposition. Plot the analyses on a single sheet and draw dotted lines tangent to the outermost deviations as in Fig. 6. Usually the curves cross each other without apparent system; failure to do so may indicate rapid regional changes or the presence of some cyclical variations of considerable magnitude. In a regional study two analyses are probably significantly different if their graphs show differences in position greater than the divergence between the dotted lines.

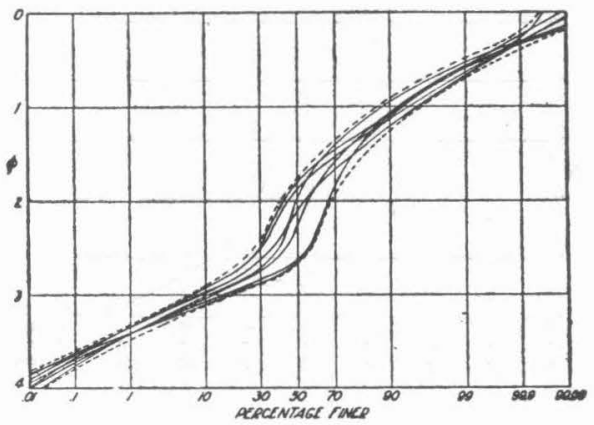

FIG. 6.-Sieve analysis of eight closely spaced samples of beach sand from Daytona Beach, Florida. The area between dotted lines indicates the amount of variability assignable to local sampling errors and laboratory errors combined.

10. If a frequency function is found that satisfactorily represents mechanical composition where complicating environmental factors are fewest, examine all poorly represented analyses for specific environmental factors capable of causing the deviations. Thus, in Fig. 2, the theoretical curve agrees well with the plotted curve except for the finest 5 per cent and the coarsest .5 per cent. From other dune-sand analyses, it appears that two terms of a Gram-Charlier series give a close approximation to the frequency function if the sand consists entirely of quartz and feldspars and if the samples are from one sedimentation unit.

The sample used in Fig. 2 was collected from the full thickness of one sedimentation unit. The coarsest grade contained only thin, weathered biotite flakes; other grades contained quartz and feldspars with subordinate mica. During trans- 
portation, thin mica flakes behave like quartz particles of smaller sieve diameter. Consequently, the sieve analysis shows coarser diameters than the theoretical analysis indicates. The sand was dusty when collected, for it was derived from nearby Whitewater Wash and was deposited in the lee of a rock hill. During moments when wind velocities are too weak to transport sand, silt settles between the interstices of the sand grains

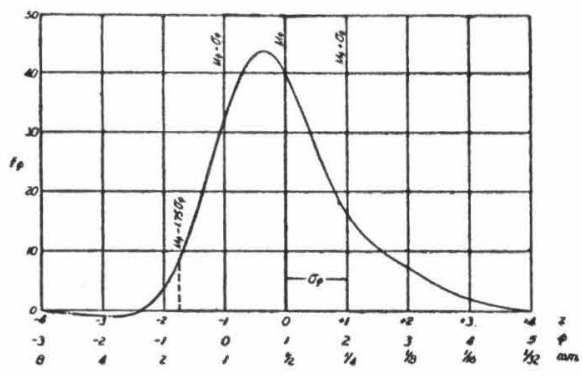

Fig. 7.-Graph of

$$
\begin{aligned}
& f(\phi)=\frac{100}{\sigma_{\phi} \sqrt{2} \pi} e^{-\frac{1}{2}\left\{\left(\phi-M_{\phi}\right) / \sigma_{\phi}\right\}^{2}} \\
& \cdot\left[1-\frac{k_{\phi}}{2}\left\{\frac{\phi-M_{\phi}}{\sigma_{\phi}}-\frac{1}{3}\left(\frac{\phi-M_{\phi}}{\sigma_{\phi}}\right)^{3}\right\}\right]
\end{aligned}
$$

when $M_{\phi}=1, \sigma_{\phi}=1, k_{\phi}=+1$.

This curve is the derivative of curve 1 in Fig. 4.

and is buried by sand transported over the hill during succeeding gusts of wind. Consequently, the sand is a mixture of two sediments, dune sand and loess, with the former greatly predominating, so that very fine sand and silt are present in greater amounts in the sample than in the dune sand alone. Another factor of similar tendency is the coating of fine silt weakly cemented to most sand grains. Such cementation occurred as the wash dried up, thus increasing the grain size, the relative effect being much greater on the finer grains. During sieving, many of these particles were knocked off and collected in the pan, thereby making the pan percentage greater than expected.

The purpose of step 10 is to systematize knowledge of secondary character- istics of the analysis and to help locate environmental factors which otherwise might be ignored as "unimportant." Emphasis here is on a specific mathematical type of curve deduced from a comparison of many analyses, whereas in step three no assumptions are made concerning the frequency function which represents the analyses, the object being to ascertain the geometric form of the basic type of curve and to test the type selected by inquiring whether deviations from it can be explained in terms of environment alone.

\section{ACKNOWLEDGEMENTS}

The writer wishes to acknowledge the assistance and suggestions of members of the staff of the Cooperative Laboratory, particularly computing by $M$. H. Duer and G. Osborne and drafting by R. W. Lindberg. A grant from the Works Progress Administration made possible a more thorough investigation than could otherwise have been made. Professors I. Campbell, F. J. Converse, W. Houston and H. J. Fraser of the California Institute of Technology and Dr. A. E. Brandt of the Soil Conservation Service read and criticised the manuscript.

\section{APPENDIX}

Principles on which the graph paper is based. In the following expression $F(\phi)$ gives the percentage of particles whose diameters lie between diameters $M_{\phi}$ and $\phi$ :

$$
\begin{gathered}
F(\phi)=\int_{M_{\phi}}^{\phi} \frac{100}{\sigma_{\phi} \sqrt{2 \pi}} e^{-\frac{1}{2}\left\{\left(\phi-M_{\phi}\right) / \sigma_{\phi}\right\}^{2}} \\
{\left[1-\frac{k_{\phi}}{2}\left\{\frac{\phi-M_{\phi}}{\sigma_{\phi}}-\frac{1}{3}\left(\frac{\phi-M_{\phi}}{\sigma_{\phi}}\right)^{3}\right\}\right] d \phi .}
\end{gathered}
$$

The notation and convention concerning the sign of $k_{\phi}$ are given on pages 5 and 9 . Figure 7 shows the graph of the frequency function, $\frac{d F(\phi)}{d \phi}$, for $M_{\phi}=1, \sigma_{\phi}=1$, and $k_{\phi}=+1$. By substituting a new variable for $\phi$,

$$
z=\frac{\phi-M_{\phi}}{\sigma_{\phi}} \text { then } d_{\phi}=\sigma_{\phi} d z
$$

the above integral takes the following form, 
for which tables are available:

$$
\begin{aligned}
& F(\phi)=\frac{100}{\sqrt{2 \pi}} \int_{0}^{z} e^{-3 z^{2}} d z \\
&-\frac{100 k_{\phi}}{6 \sqrt{2 \pi}}\left[1-\left(1-z^{2}\right) e^{-\frac{1}{z} z^{2}}\right] .
\end{aligned}
$$

The first term is the integral of the normal curve of error; when $k_{\phi}=0$, the second term vanishes.

By means of a table for the first term and elementary calculus for the second, it is readily shown that the percentage of particles finer than $M_{\phi}$ is $50-6.649 k_{\phi}$ and that the percentage of particles finer than $M_{\phi}$ but coarser than $M_{\phi}+\sigma_{\phi}$ is $34.134-6.649 k_{\phi}$. The percentage finer than $M_{\phi}+\sigma_{\phi}$ (fig. 7) is then:

$\left(50-6.649 k_{\phi}\right)-\left(34.134-6.649 k_{\phi}\right)=15.866$. Therefore, the cumulative percentage at one phi standard deviation finer than the phi arithmetic mean diameter will always be 15.866 per cent, regardless of the value of the skewness, if two terms of a Gram-Charlier series represent the analysis. Similarly the percentage finer than diameter $M_{\phi}-\sigma_{\phi}$ is always 84.134 per cent.

These two cumulative percentages, $\mathbf{1 5 . 8 6 6}$ and 84.134, are loci of intersection of the family of curves formed from (3) by varying $k_{\phi}$ while $M_{\phi}$ and $\sigma_{\phi}$ are held constant. Application of the principles of analytical geometry to equation (2) shows that varying the value of $M_{\phi}$ affects merely the relative position of the curve on the graph (fig. 4, curves 1 and 4). Variations in $\sigma_{\phi}$ affect the scale of the graph (fig. 4, curves 1 and 2). Only variations in $k_{\phi}$ cause changes in the shape of the curve. These statements are independent of the kind of graph used, provided particle diameter is expressed in phi units.

Construction of the lines of the graph and the diameter scales. The vertical phi scales and horizontal lines of the graph are ordinary equal interval scales. The corresponding logarithmic millimeter scales are constructed as described by Croxton and Cowden (2, pp. 105-107). The vertical, cumulative percentage lines are so located that the integral in the first term of (3) will plot a straight line. This is accomplished by spacing the percentage lines proportional to their corresponding $z$ values (7). In other words, each percentage line (denoting area under the normal curve) is spaced proportional to the numerical value of its abscissa.

In a graph extending from .01 to 99.99 per cent, the median or 50 per cent line lies midway between; furthermore, the lines on the left half of the graph are mirror images of those on the right half. Pearson (15, pp. 2-10) gives a table showing the values of the $a b-$ scissae of the normal curve as a function of the frequency or cumulative percentage expressed as a decimal. In this table the abscissa is denoted by $x$ and the cumulative percentage by $\frac{1}{2}\left(1+\alpha_{x}\right)$.

The $z$ value corresponding to 99.99 per cent is 3.719. For a graph 28.5 inches wide, one $z$ unit is represented by a length of $14.25 / 3.719=3.832$ inches. Since the $z$ value for the 80 per cent line is .842 , it is located $.842 \times 3.832$ inches to the right of the 50 per cent line. The 20 per cent line is the same distance to the left. Similar computations are made for each pair of lines.

Theory and construction of the phi standard deviation scale. When equation (1) represents the analysis, it has been shown that there are two loci, located at 15.9 and 84.1 cumulative per cent, which are always one standard deviation on either side of $M_{\phi}$, regardless of the value of $k_{\phi}$. On the graph the vertical distance between these points, measured by the distance between their intercepts on the phi scale, is thus equal to two standard deviations. Consequently, if a $\sigma_{\phi}$ scale were laid out along the 15.9 per cent line starting at the horizontal line $\phi=0$, the values of $\sigma_{\phi}$ and the spacings would be identical with those of the regular $\phi$ scale. Since the phi standard deviation is proportional to the tangent of the slope of the line connecting the two loci, the scale can be transferred to any convenient line by means of similar triangles.

In constructing the scale, points are located on the 15.9 and 84.1 per cent lines at a distance equal to two phi units below and above the horizontal line passing through the small circle. A line is drawn through these points intersecting the vertical line selected for the standard deviation scale. The distance from the horizontal line to this intersection is then divided into the correct number of divisions and the scale is drawn radially, using the small circle as a center.

Because this construction fixes the origin, 
it is necessary always to translate the origin from the observed mean diameter to the circle by drawing the line $E F$ parallel to $A B$ (fig. 2).

Theory of the determination of $M_{\phi}$. When $k_{\phi}$ is zero, the phi median and the phi arithmetic mean diameter coincide, so that both are obtained from the intersection of the straight line graph with the $\mathbf{5 0}$ per cent line. Since the family of curves formed by varying the skewness while $M_{\phi}$ and $\sigma_{\phi}$ are held constant have two points of common intersection, the straight line graph when $k_{\phi}$ is zero includes these points. Consequently, to obtain $M_{\phi}$ when $k_{\phi}$ is not zero, simply draw a straight line through the loci at 15.9 and 84.1 cumulative per cent and determine $M_{\phi}$ as above.

Theory and construction of the skewness scale. When equation (1) represents the analysis, the curvature of the graph is caused by the skewness alone. Consequently, the skewness can be measured by determining the cumulative percentage at which the graph crosses some multiple of the standard deviation, $z$, provided $z$ is not equal to 1 . Values of $z$ are measured along the vertical scale of the graph starting at $M_{\phi}$ and expressed as mul- tiples of the standard deviation in accordance with equation (2). For reliable determination of the skewness, the extreme ends of the distribution are unsatisfactory on account of sampling errors. Values near $z= \pm 1$ are too insensitive. A $z$ value of 1.75 gives a good compromise and conveniently corresponds almost exactly to 4 and 96 cumulative per cent for the normal curve. Since the spacings of the vertical percentage lines are proportional to their corresponding normal curve $z$ values, it follows from the geometry of the right triangle that the distance $G H$ on figure 2 is 1.75 times the scale value of one standard deviation. The lines $D I$ and $I J$ merely enable this value of $1.75 z$ to be measured from $M_{\phi}$. The percentage finer than $M_{\phi}-1.75 \sigma_{\phi}$ is equal to the percentage finer than $M_{\phi}$ plus the percentage between $M_{\phi}$ and $M_{\phi}-1.75 \sigma_{\phi}$; from equation (3) these values are: $\left(50-6.649 k_{\phi}\right)+\left(45.994+9.615 k_{\phi}\right)$ $=95.994+2.966 k_{\phi}$.

By substituting values of the skewness, the corresponding percentages are found which are then marked off to form the skewness scale.

\section{REFERENCES}

1. CAMP, B. H., 1934, The mathematical part of elementary statistics, D. C. Heath Co.

2. Croxton, F. E., and Cowden, D. J., 1934, Practical business statistics, Prentice-Hall, Inc.

3. Fisher, R. A., 1932, Statistical methods for research workers, Oliver and Boyd.

4. Goldman, F. H., and Dalla Valle, J. M., 1939, An accurate method for the determination of the components of a heterogeneous particulate mineral system: Am. Mineralogist, vol. $24,40-47$.

5. Hatch, T., and ChoAte, S. P., 1929, Statistical description of the size properties of nonuniform particulate substances: Franklin Inst. Jour., vol. 207, 369-387.

6. HAтch, T., 1933, Determination of average size from the screen analysis of non-uniform particulate substances: Franklin Inst. Jour., vol. 215, 27-37.

7. Hazen, A., 1914, Storage to be provided in impounding reservoirs: Am. Soc. Civil Engrs., Trans., vol. $77,1539-1669$, especially $1664-1668$.

8. Loveland, R. P., and Trivelli, A. P., 1927, Mathematical methods of frequency analysis of size of particles, Part I, methods: Franklin Inst., Jour., vol. 204,-193-217.

9. KRUMBEIN, W. C., 1936, The use of quartile measures in describing and comparing sediments: Am. Jour. Sci., vol. 32, 98-111.

10. -1934 , Size frequency distribution of sediments: J. Sed. Pet., vol. 4, 65-77.

11. - 1936, Application of logarithmic moments to size frequency distribution of sediments: J. Sed. Pet., vol. 6, 35-47.

12. 1938 , Size frequency distributions of sediments and the normal phi curve: J. Sed. Pet., vol. 8, 84-90.

13. Krumbein, W. C., and Petrijohn, F. J., 1938, Manual of sedimentary petrography, D. Appleton-Century Co.

14. Oтто, H., 1938, The sedimentation unit and its use in field sampling: Jour. Geology., vol. $46,569-582$.

15. Pearson, K., 1932, Tables for statisticians and biometricians, Part II, Cambridge University Press.

16. Rouse, H., 1938, Fluid mechanics for hydraulic engineers, McGraw-Hill Book Company. 17. Wadell, H., 1934, Some new sedimentation formulas: Physics, vol. 5, 281-291. 